\title{
Article
}

\section{Research on the Decision-Making Mechanism of Livestreaming E-commerce Supply Chain Based on Three-Party Evolutionary Game}

\author{
Jixiao $\mathrm{Wu}^{1, *}$, Yinghui Wang ${ }^{2}$ \\ 1 Faculty of Economics and Management, Shijiazhuang Tiedao University, Shijiazhuang, 050043, China; \\ 1024985523@qq.com \\ 2 Faculty of Economics and Management, Shijiazhuang Tiedao University, Shijiazhuang, 050043, China; \\ wang112111@163.com \\ * Correspondence: 1024985523@qq.com
}

\begin{abstract}
With the diversified development of media forms, livestreaming e-commerce has become a new sales model. Unlike the traditional sales model, this paper constructs a three-party game model composed of manufacturers, social media influencers and consumers based on the livestreaming model. It explores the equilibrium strategy selections of each participant in the supply chain system. In analyzing the evolutionary game stability of each participant, this paper obtains the equilibrium strategy and stability factors by analysis of the income and expenditure matrix. It uses the simulation model to analyze reasons for strategy selections of different game participants in the livestreaming e-commerce model. The results show that the strategies of manufacturers, social media influencers and consumers' selections have different impacts on their decision-making mechanism, and manufacturers are more sensitive to consumers' active participation in livestreaming ecommerce.
\end{abstract}

Keywords: livestreaming e-commerce; evolutionary game; multi-party behavior; replicator dynamics equation; three-party game theory

\section{Introduction}

With the promotion of the "Internet Plus" policy and the implementation of information engineering in China, the sales form of "livestreaming + e-commerce" has been favoured by more consumers: according to the 46th "Statistical Report on China's Internet Development" released by China Internet Network Information Center, as of June 2020, the number of Chinese netizens has reached 940 million, with an Internet penetration rate of 67.0 per cent; among them, the number of livestreaming e-commerce users has reached 309 million, an increase of 44.3 million compared to March 2020, with a scale growth rate of 16.7 per cent. The scale of online retail users reached 749 million, accounting for 79.7 per cent of the total netizens. Online shopping has become consumers' primary shopping method.

Livestreaming e-commerce is different from gift-giving in livestreaming. It is a new shopping model in which social media influencers use live broadcast technology for online products display, consultation, and shopping guidance through livestreaming platforms. It is mainly divided into two models: merchants directly promote their products, and professional social media influencers recommend products [1]. Compared with the traditional sales model, livestreaming e-commerce has the following characteristics: (1) Livestreaming e-commerce is endowed with more vital interaction and affinity. Consumers can directly communicate with merchants or social media influencers on livestreaming platforms to communicate and bargain. (2) Livestreaming e-commerce bypasses the traditional channels of middlemen and realizes communications between products and consumers to achieve "the lowest price of the entire network" of products and establish a price 
advantage. (3) Livestreaming e-commerce depends on influencers' charm. It is essentially an advertising campaign prepared by influencers for consumers through livestreaming platforms. It is necessary to adopt specific mutually beneficial means to provide a discount for consumers to purchase products through actively responding to influencers' calls.

Social media influencers are closely related to livestreaming e-commerce. From 2015 to 2018 , the compound growth rate of China's social media influencers industry reached 59.4 per cent, and the industrial scale in 2018 exceeded 100 billion RMB [2]. Social media influencers let more users participate in livestreaming active decision-making, to improve users' satisfaction [3-5]. Users can motivate influencers by gift-giving to improve their enthusiasm for livestreaming [6-8]. Influencers can improve the number of users by optimizing livestreaming e-commerce and enhancing their social influence $[5,9]$. However, at present, the academic research on livestreaming mainly focuses on live content and giftgiving. Although the data is rich and the research is detailed, the degree of agreement with livestreaming e-commerce is low, and there is little direct research on the literature on livestreaming e-commerce. Cunningham analyzed the platforms, policies, and difficulties faced by China's webcasting and pointed out that social media influencers can successfully drive economic development, meet marketing needs, and guide consumers to shift from a traditional sales model to a livestreaming sales model [10]. This benefit can be expanded through the livestreaming e-commerce model to enhance consumers' desire to buy products [7, 11-14]. But the research on influencers focuses on their economic benefits or social impact, only a little research on influencers income and expenditure.

Livestreaming e-commerce is based on social media influencers as the spokesperson and the number of influencers' fans as the leading factor [14]. By gathering popularity on livestreaming platforms and social media, relying on the huge fan group to sell products, livestreaming e-commerce is transforming the fans into purchasing power [15, 16]. It plays an important role in expanding sales markets and enhancing brands [17]. However, the benefits of livestreaming e-commerce depend on the enthusiasm of influencers and consumers [18]. Therefore, it's necessary to clarify the income model [19, 20], design a reward and punishment mechanism [21], and explore the mutual influence [22]. The supply chain includes manufacturers, social media influencers, and consumers, which involves multiparty decision-making, so game theory optimization is selected [23-29]. At present, scholars have some research on the three-party game [30-32], but few scholars combine the three-party game with the situation of the income and expenditure of livestreaming ecommerce. To better analyze the relationship among manufacturers, social media influencers, and consumers in the livestreaming e-commerce model, this paper adopts game theory to explore the decision-making evolution mechanism among supply chain participants based on previous research.

To better analyze the decision-making mechanism of the livestreaming e-commerce supply chain, this paper makes innovations in the following aspects: (1) Integrate livestreaming e-commerce with the online supply chain to explore the supply chain income and expenditure in the livestreaming e-commerce model. Analyze the benefits of the supply chain based on the three-party game model to explore the decision-making evolution process of manufacturers, social media influencers, and consumers in the livestreaming e-commerce supply chain. (2) At present, little literature involves the income and expenditure of social media influencers in livestreaming e-commerce. This paper takes social media influencers as the main participant of the game. It analyzes livestreaming e-commerce from influencers' perspective to facilitate influencers to make decisions and choices. (3) It analyzed the income and expenditure of livestreaming e-commerce and found the equilibrium point of the participants in the livestreaming e-commerce model. It is conducive to explore the phenomenon of livestreaming e-commerce further and promoting the development of livestreaming e-commerce. 


\section{Methods}

\subsection{Problem description}

The supply chain involved in this paper is composed of manufacturers, social media influencers, and consumers, with livestreaming e-commerce as the main innovative model. Livestreaming e-commerce involved in this paper is operated by professional influencers who specifically promote products. Consumers are an important part of livestreaming e-commerce. As a participant who watches livestreaming and purchases products, consumers will receive certain discounts during watching livestreaming e-commerce. When carrying out livestreaming e-commerce, manufacturers and influencers need to clarify each other's rights and obligations in the form of contracts and pay some input costs by the contracts to guarantee to carry out livestreaming e-commerce.

Manufacturers, social media influencers, and consumers are the three participants in the livestreaming e-commerce supply chain. Consumers mainly benefit from influencers and manufacturers by rewarding influencers and purchasing products from manufacturers, thereby promoting influencers and manufacturers' preparations for livestreaming ecommerce. Manufacturers are the product providers in the supply chain. They manufacture products according to the number of orders received by influencers on livestreaming platforms and improve the products' quality to satisfy consumers' demands. Social media influencers involved in this paper refers to those general influencers who do not have large numbers of fans. They promote products provided by manufacturers on livestreaming platforms through livestreaming e-commerce and increase consumers' desire to purchase products to increase product sales. In the game among manufacturers, social media influencers, and consumers, the three participants are rational in the game process. They will find optimal strategies for each other through multiple games. The internal distribution mechanism and evolutionary effect game model of the participants of the livestreaming e-commerce supply chain are shown in Figure 1.

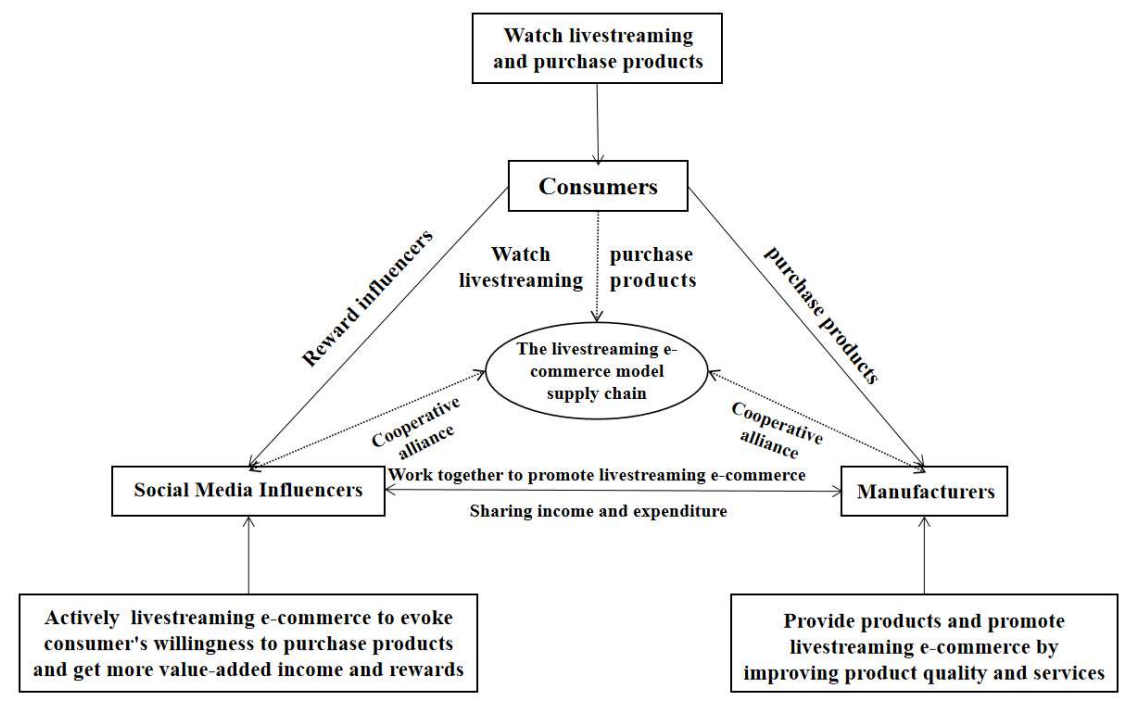

Figure 1. Game model of manufacturers, social media influencers, and consumers in the livestreaming e-commerce model.

After referring to the literature of other scholars, this paper defines the strategy selection behavior of social media influencers, manufacturers and consumers in this game model as social media influencers have two strategies to choose from: (1) Actively carry out livestreaming e-commerce by increasing investment in influencers' charm, innovating livestreaming strategies, increasing the number of fans, and analyzing fans' loyalty to make more profits. (2) Conservatively carry out livestreaming e-commerce by simplifying 
livestreaming procedures, reducing strategic innovation, relying on existing fans, and not paying attention to changes in the number of fans to save the cost of carrying out livestreaming e-commerce. Manufacturers have two strategies to choose from: (1) Actively optimize products by enhancing supply chain efficiency, reducing logistics time, and improving service quality to obtain more profits. (2) Conservatively deal with the products by reducing quality supervision costs, cutting logistics costs, and reducing aftersales service input. Consumers have two strategies to choose from: (1) Actively respond to influencers' calls and increase purchases. (2) Conservatively deal with influencers' calls and reduce purchases.

As main participants, manufacturers and social media influencers need to invest in particular human, material, and financial resources in livestreaming e-commerce. The total cost of investing in these resources is $I$. When consumers actively respond to influencers' calls to purchase products, it will reduce the total cost I paid by manufacturers and influencers. The reduced cost is $J$. Now the total cost paid by manufacturers and influencers is $(I-J)$. The cost-sharing ratio coefficient between manufacturers and influencers is $n$, the cost paid by manufacturers is $n I$ or $n(I-J)$, and the cost paid by influencers is (1-n)I or $(1-n)(I-J)$.

Consumers who actively respond to social media influencers' calls to purchase products receive A. $\theta$ represents the percentage of discount obtained when consumers choose a conservative strategy to the discount obtained when consumers choose an active strategy. When consumers choose a conservative strategy to purchase products, the discount they get is $\theta A$. When both manufacturers and social media influencers choose an active strategy, the value-added income obtained is $D$. The sharing ratio coefficient of $D$ is $m$, which shows the value-added income of manufacturers in livestreaming e-commerce is $m D$. The value-added income of social media influencers in livestreaming e-commerce is (1-m) $D$. When social media influencers actively carry out livestreaming e-commerce but manufacturers conservatively deal with products, manufacturers can obtain some freeriding income $E$. When manufacturers actively optimize products but social media influencers conservatively carry out livestreaming e-commerce, influencers can obtain freeriding income $F$. In addition, consumers will give some reward fee $H$ to social media influencers who actively carry out livestreaming e-commerce.

Manufacturers and social media influencers from breaching contracts during livestreaming e-commerce, when manufacturers actively optimize products, but influencers conservatively carry out livestreaming e-commerce, influencers need to pay some compensation fee $W$ to manufacturers according to agreements. When social media influencers actively carry out livestreaming e-commerce but manufacturers conservatively deal with products, manufacturers need to pay $K$ to influencers according to agreements.

The specific game parameters of manufacturers, social media influencers, and consumers in the livestreaming e-commerce model is shown in Table 1.

Table 1. Game parameters of manufacturers, social media influencers, and consumers in the livestreaming e-commerce model.

\begin{tabular}{cc}
\hline Parameter & Description \\
\hline$\alpha$ & Probability of consumers actively responding to influencers' calls to purchase products \\
$\gamma$ & Probability of manufacturers choosing to optimize products actively \\
$A$ & Probability of influencers choosing to carry out livestreaming e-commerce actively \\
$\theta$ & The percentage of discounts that consumers get when choosing a conservative strategy as compared \\
& Value-added income obtained by manufacturers and social media influencers in livestreaming e- \\
$D$ & commerce when both choose active strategies \\
\hline
\end{tabular}




\begin{tabular}{|c|c|}
\hline$m$ & Coefficient of apportionment ratio of value-added income obtained by manufacturers \\
\hline$E$ & $\begin{array}{l}\text { The free-riding income obtained by manufacturers when influencers actively carry out livestreaming } \\
\text { e-commerce, but manufacturers conservatively deal with products }\end{array}$ \\
\hline$F$ & $\begin{array}{l}\text { The free-riding income obtained by social media influencers when manufacturers actively optimize } \\
\text { products but influencers conservatively carry out livestreaming e-commerce }\end{array}$ \\
\hline$H$ & Cost of rewarding influencers who actively carry out livestreaming e-commerce by consumers \\
\hline$I$ & Cost invested in by manufacturers and influencers when they prepare for livestreaming e-commerce \\
\hline$J$ & Reduced input costs when consumers respond actively \\
\hline$n$ & Manufacturer's cost apportionment ratio coefficient \\
\hline$W$ & $\begin{array}{l}\text { Compensation paid by social media influencers to manufacturers when manufacturers actively opti- } \\
\text { mize products but influencers conservatively carry out livestreaming e-commerce }\end{array}$ \\
\hline K & $\begin{array}{l}\text { Compensation paid by manufacturers to social media influencers when influencers actively carry out } \\
\text { livestreaming e-commerce, but manufacturers conservatively deal with products }\end{array}$ \\
\hline
\end{tabular}

\subsection{Construction of Income and Expenditure Matrix}

In this game model, manufacturers, social media influencers, and consumers make strategic selections based on probabilities. It is assumed that the probability of consumers actively responding to influencers' call to purchase products is $\alpha$. The probability of conservatively coping with influencers' call to purchase products is $(1-\alpha)$. The probability of manufacturers choosing to optimize products actively is $\beta$. The probability of manufacturers choosing to deal with products conservatively is $(1-\beta)$. The probability of influencers choosing to carry out livestreaming e-commerce actively is $\gamma$. The probability of influencers choosing to carry out livestreaming e-commerce conservatively is $(1-\gamma) . \alpha, \beta$, $\gamma \in[0,1]$. Based on previous research [33-35] and combined with the above conditions, the income and expenditure matrix of the three-party game of manufacturers, social media influencers, and consumers in the livestreaming e-commerce model are shown in Table 2.

Table 2. The income and expenditure matrix of the three-party game of manufacturers, social media influencers, and consumers in the livestreaming e-commerce model.

\begin{tabular}{|c|c|c|c|c|}
\hline & \multicolumn{2}{|c|}{$\begin{array}{l}\text { Consumers actively respond to influencers' call to purchase } \\
\text { products }(\alpha)\end{array}$} & \multicolumn{2}{|c|}{$\begin{array}{l}\text { Consumers conservatively deal with influencers' call to pur- } \\
\text { chase products (1- } \alpha)\end{array}$} \\
\hline & $\begin{array}{c}\text { influencers actively carry } \\
\text { out livestreaming e-com- } \\
\text { merce }(\gamma)\end{array}$ & $\begin{array}{l}\text { influencers conservatively } \\
\text { carry out livestreaming e- } \\
\text { commerce }(1-\gamma)\end{array}$ & $\begin{array}{c}\text { influencers actively carry } \\
\text { out livestreaming e-com- } \\
\text { merce }(\gamma)\end{array}$ & $\begin{array}{l}\text { influencers conservatively } \\
\text { carry out livestreaming e-com- } \\
\text { merce }(1-\gamma)\end{array}$ \\
\hline Manufacturers ac- & $A-H$ & $A$ & $\theta A$ & $\theta A$ \\
\hline tively optimize prod- & $m D-n(I-J)$ & $W-n(I-J)$ & $m D-n I$ & $-n l+W$ \\
\hline ucts $(\beta)$ & $(1-m) D-(1-n)(I-J)+H$ & $F-W$ & $(1-m) D-(1-n) I$ & $F-W$ \\
\hline Manufacturer conser- & $A-H$ & $A$ & $\theta A$ & $\theta A$ \\
\hline vatively deal with & $E-K$ & 0 & $E-K$ & 0 \\
\hline products (1- $\beta)$ & $-(1-n)(I-J)+K+H$ & 0 & $-(1-n) I+K$ & 0 \\
\hline
\end{tabular}

In Table 2, the manufacturers' participation restriction is reflected in the compensation $K$ paid when the contract is violated. The incentive restriction is reflected in the benefit distribution coefficient $m$ of the income from the promotion of livestreaming e-commerce. The influencers' participation restriction in livestreaming e-commerce is reflected in $W$ paid when the contract is violated. The incentive restriction is reflected in the benefit 
distribution coefficient (1- $m$ ) of the income from the promotion of livestreaming e-commerce.

\section{Evolutionary Stability Strategy Solution}

\subsection{Consumers' Expected Income and Expenditure}

The expected income and expenditure when consumers actively respond to social media influencers' calls to purchase products are:

$$
\pi_{c 1}=\beta \gamma(A-H)+\beta(1-\gamma) A+(1-\beta) \gamma(A-H)+(1-\beta)(1-\gamma) A
$$

The expected income and expenditure when consumers conservatively deal with influencers' calls to purchase products are:

$$
\pi_{c 2}=\beta \gamma \theta A+\beta(1-\gamma) \theta A+(1-\beta) \gamma \theta A+(1-\beta)(1-\gamma) \theta A
$$

The expected income and expenditure of consumers in the livestreaming e-commerce model are:

$$
\pi_{c}=\alpha \pi_{c 1}+(1-\alpha) \pi_{c 2}
$$

According to the income and expenditure matrix, the replicator dynamics equation of consumers when actively responding to influencers' calls to purchase products are:

$$
F(\alpha)=\alpha\left(\pi_{c 1}-\pi_{c}\right)=\alpha(1-\alpha)\left(\pi_{c 1}-\pi_{c 2}\right)=\alpha(1-\alpha)[(1-\theta) A-\gamma H]
$$

\subsection{Manufacturers' Expected Income and Expenditure}

The expected income and expenditure when manufacturers actively optimize products are:

$$
\pi_{m 1}=\alpha \gamma[m D-n(I-J)]+\alpha(1-\gamma)[-n(I-J)+W]+(1-\alpha) \gamma(m D-n I)+(1-\alpha)(1-\gamma)(-n I+W)
$$

The expected income and expenditure when manufacturers conservatively deal with products are:

$$
\pi_{m 2}=\alpha \gamma(E-K)+(1-\alpha) \gamma(E-K)
$$

The expected income and expenditure of manufacturers in the livestreaming e-commerce model are:

$$
\pi_{m}=\beta \pi_{m 1}+(1-\beta) \pi_{m 2}
$$

According to the income and expenditure matrix, the replicator dynamics equation of manufacturers when actively optimizing products are:

$$
F(\beta)=\beta\left(\pi_{m 1}-\pi_{m}\right)=\beta(1-\beta)\left(\pi_{m 1}-\pi_{m 2}\right)=\beta(1-\beta)[W-n I+o n J+\gamma(m D-W-E+K)]
$$

\subsection{Social Media Influencers' Expected Income and Expenditure}

The expected income and expenditure when social media influencers actively carry out livestreaming e-commerce are:

$$
\pi_{i 1}=\alpha\{(1-m) D-(1-n)(I-J)+H]+(1-\alpha) \beta[(1-m) D-(1-n) I]+\alpha(1-\beta)[-(1-n)(I-J)+K+H]+(1-\alpha)(1-\beta)[-(1-\mathrm{n}) I+K]
$$

The expected income and expenditure when influencers conservatively carry out livestreaming e-commerce are:

$$
\pi_{i 2}=\alpha \beta(F-W)+(1-\alpha) \beta(F-W)
$$


The expected income and expenditure of influencers in the livestreaming e-commerce model are:

$$
\pi_{i}=\gamma \pi_{i 1}+(1-\gamma) \pi_{i 2}
$$

According to the income and expenditure matrix, the replicator dynamics equation of social media influencers when actively carrying out livestreaming e-commerce are:

$$
F(\gamma)=\gamma\left(\pi_{i 1}-\pi_{i}\right)=\gamma(1-\gamma)\left(\pi_{i 1}-\pi_{i 2}\right)=\gamma(1-\gamma)\{K-(1-n) I+\alpha[(1-n) J+H]+\beta[(1-m) D-K+W-F]\}
$$

According to the method proposed by Friedman, the evolutionary stability strategy (ESS) of the differential equation system can be obtained from the local stability analysis of the Jacobian matrix of the system, and the Jacobian matrix of the system can be obtained from equations (4), (8), (12) as:

$$
M=\left[\begin{array}{ccc}
(1-2 \alpha)[(1-\theta) A-\gamma H] & 0 & -\alpha(1-\alpha) H \\
\beta(1-\beta) n J & (1-2 \beta)[W-n I+\alpha n J+\gamma(m D-W-E+K)] & \beta(1-\beta)(m D-W-E+K) \\
\gamma(1-\gamma)[(1-n) J+H] & \gamma(1-\gamma)[(1-m) D-K+W-F] & (1-2 \gamma)\{K-(1-n) I+\alpha[(1-n) J+H]+\beta[(1-m) D-K+W-F]\}
\end{array}\right]
$$

On the basis of replicator dynamics equations of the above-mentioned game groups, when setting $X=\left(\frac{d F(\alpha)}{d t}, \frac{d F(\beta)}{d t}, \frac{d F(\gamma)}{d t}\right)^{T}=f(X, t)=0$, the local Nash equilibrium points in the three-party game system are obtained as: $X_{1}=(0,0,0), X_{2}=(0,1,0), X_{3}=(0,0,1), X_{4}=(0,1,1)$, $X_{5}=(1,0,0), \quad X_{6}=(1,0,1), \quad X_{7}=(1,1,0), \quad X_{8}=(1,1,1), \quad X_{9}=\left(a^{*}, \beta^{*}, \gamma^{*}\right)=\left(\frac{(1-\theta)(E-K+W-m D)-H W+n H I}{n H J}\right.$, $\left.\frac{(1-\theta) A(H+J-n J)(E-K+W-m D)+H(n H I+n J K+n J W-H W-W J)}{n H J(F-D+K-W+m D)}, \frac{(1-\theta) A}{H}\right)$. The eight equilibrium points $X_{1} \sim X_{8}$ constitute the boundary of the evolutionary game. According to the evolutionary game theory, when all eigenvalues of the Jacobian matrix are non-positive, the equilibrium point of the game is the evolutionary stable point of the system (ESS).

\subsection{Stability Analysis of Equilibrium Points}

When the equilibrium point $X_{1}=(0,0,0)$, the Jacobian matrix is:

$$
M_{1}=\left[\begin{array}{ccc}
(1-\theta) A & 0 & 0 \\
0 & W-n I & 0 \\
0 & 0 & K-(1-n) I
\end{array}\right]
$$

At this point, the characteristic value of the Jacobian matrix is $\lambda_{1}=(1-\theta) A$, $\lambda_{2}=W-n I, \quad \lambda_{3}=K-(1-n) I$. It can be known from the same principle that by substituting eight equilibrium points into the Jacobian matrix (13), the eigenvalues of the Jacobian matrix corresponding to the equilibrium points can be obtained, as shown in Table 3.

Table 3. Eigenvalues of the Jacobian matrix.

\begin{tabular}{cccc}
\hline Equilibrium point & Eigenvalues $\lambda_{1}$ & Eigenvalues $\lambda_{2}$ & Eigenvalues $\lambda_{3}$ \\
\hline$X_{1}(0,0,0)$ & $(1-\theta) A$ & $W-n I$ & $K-(1-n) I$ \\
$X_{2}(0,1,0)$ & $(1-\theta) A$ & $n I-W$ & $(1-m) D-(1-n) I+W-F$ \\
$X_{3}(0,0,1)$ & $(1-\theta) A-H$ & $m D-n I-E+K$ & $(1-n) I-K$ \\
$X_{4}(0,1,1)$ & $(1-\theta) A-H$ & $n I-m D+E-K$ & $(1-n) I-(1-m) D+F-W$ \\
$X_{5}(1,0,0)$ & $-(1-\theta) A$ & $W-n(I-J)$ & $H-(1-n)(I-J)+K$ \\
\hline
\end{tabular}




\begin{tabular}{cccc}
\hline$X_{6}(1,0,1)$ & $H-(1-\theta) A$ & $m D-n(I-J)-E+K$ & $(1-n)(I-J)-H-K$ \\
$X_{7}(1,1,0)$ & $-(1-\theta) A$ & $n(I-J)-W$ & $(1-m) D-(1-n)(I-J)+H+W-E$ \\
$X_{8}(1,1,1)$ & $H-(1-\theta) A$ & $n(I-J)-m D+E-K$ & $(1-n)(I-J)-(1-m) D-H-W+E$ \\
\hline
\end{tabular}

To facilitate the analysis of the symbols of the eigenvalues corresponding to different Nash equilibrium points without loss of generality, this paper assumes $(1-\theta) A-H>0, m D$ $n I-E+K>0$ and $(1-m) D-(1-n) I+W-F>0$. It means the net income brought by manufacturers, social media influencers, and consumers who actively promote livestreaming e-commerce is greater than conservatively dealing with livestreaming e-commerce. Due to many parameters and the tedious calculations in the model, this paper discusses the stability strategy of the evolutionary game in the following three scenarios.

\subsubsection{Scenario 1}

When $H-(1-n)(I-J)+K<0$ and $W-n(I-J)<0$, the sum of the compensation paid to social media influencers by manufacturers when manufacturers choose a conservative strategy and the consumers' reward for influencers are less than the cost paid by influencers when consumers actively respond to influencers' calls. And the compensation paid to manufacturers by influencers when influencers choose a conservative strategy is less than the cost paid by manufacturers when consumers actively respond to influencers' calls.

Table 4 demonstrates that the eigenvalues of the Jacobian matrix corresponding to the equilibrium points $X_{5}(1,0,0)$ and $X_{s}(1,1,1)$ are non-positive, then the system has two stable points $(1,0,0)$ and $(1,1,1)$ in this scenario. The corresponding evolution strategies are (active response, conservative treatment, and conservative livestreaming) and (active response, active optimization, active livestreaming).

\subsubsection{Scenario 2}

When $K-(1-n) I>0$ and $W-n I>0$, the compensation paid to social media influencers by manufacturers when manufacturers choose a conservative strategy is more than the cost paid by influencers when consumers conservatively respond to influencers' calls. Or the compensation paid to manufacturers by influencers when influencers choose a conservative strategy is more than the cost paid by manufacturers when consumers conservatively respond to influencers' calls.

Table 4 demonstrates that the eigenvalues of the Jacobian matrix corresponding to the equilibrium point $X_{8}(1,1,1)$ are non-positive, then the system has one stable point $(1,1,1)$ in this scenario. The corresponding evolution strategy is (active response, active optimization, active livestreaming).

\subsubsection{Scenario 3}

When $H-(1-n)(I-J)+K>0$ and $K-(1-n) I<0$ or $W-n(I-J)>0$ and $W-n I<0$, the sum of the compensation paid to social media influencers by manufacturers when manufacturers choose a conservative strategy and the consumers' reward for influencers are more than the cost paid by influencers when consumers actively respond to influencers' calls. And the compensation paid to influencers by manufacturers when manufacturers choose a conservative strategy is less than the cost paid by influencers when consumers conservatively respond to influencers' calls.

Or the compensation paid to manufacturers by influencers when social media influencers choose a conservative strategy is more than the cost paid by manufacturers when consumers actively respond to influencers' calls. And the compensation paid to manufacturers by influencers when influencers choose a conservative strategy is less than the cost paid by manufacturers when consumers conservatively respond to influencers' calls.

Table 4 demonstrates that the eigenvalues of the Jacobian matrix corresponding to the equilibrium point $X_{8}(1,1,1)$ are non-positive, then the system has one stable point $(1,1,1)$ in this scenario. The corresponding evolution strategy is (active response, active optimization, active livestreaming). 
Table 4. Local stability of equilibrium points in scenarios 1, 2, 3.

\begin{tabular}{cccccccccccccc}
\hline \multirow{2}{*}{$\begin{array}{c}\text { Equilibrium } \\
\text { point }\end{array}$} & \multicolumn{9}{c}{ Scenario 1} & \multicolumn{3}{c}{ Scenario 2} \\
\cline { 2 - 10 } & $\lambda_{1}$ & $\lambda_{2}$ & $\lambda_{3}$ & Stability & $\lambda_{1}$ & $\lambda_{2}$ & $\lambda_{3}$ & Stability & $\lambda_{1}$ & $\lambda_{2}$ & $\lambda_{3}$ & Stability \\
\hline$X_{1}(0,0,0)$ & + & - &,+- & Unstable & + & + &,+ & Stable & + & - & - & Unstable \\
$X_{2}(0,1,0)$ & + & + &,+- & Stable & + & - & + & Unstable & + & + & + & Stable \\
$X_{3}(0,0,1)$ & + & + & + & Stable & + & + & - & Unstable & + & + & + & Stable \\
$X_{4}(0,1,1)$ & + & - & - & Unstable & + & - & - & Unstable & + & - & - & Unstable \\
$X_{5}(1,0,0)$ & - & - & - & ESS & - & + & + & Unstable & - & + & + & Unstable \\
$X_{6}(1,0,1)$ & - & + & + & Unstable & - & + & - & Unstable & - & + & - & Unstable \\
$X_{7}(1,1,0)$ & - & + & + & Unstable & - & - & + & Unstable & - & - & + & Unstable \\
$X_{8}(1,1,1)$ & - & - & - & ESS & - & - & - & ESS & - & - & - & ESS \\
\hline
\end{tabular}

\section{Results and Discussion}

Based on the parameters provided about other literature [33,36], this paper Assumes that in the initial game, manufacturers, social media influencers, and consumers all have a 50 per cent probability of choosing different livestreaming e-commerce strategies. This paper refers to the case of Luo Yonghao's livestreaming e-commerce of Mi Jumbo Gel Ink Pen on April 1, 2020, and sets the time step to 0.001 . The three-party game's relevant parameters of income and expenditure matrix are set as follows (unit: ten thousand RMB): Consumers get a discount $A$ of 10 for purchasing products in livestreaming e-commerce. Manufacturers and influencers choose an active strategy to get the value-added income $D$ of 70 in livestreaming e-commerce. The cost $I$ invested in by both manufacturers and influencers in the preparation of livestreaming e-commerce is 60 . The free-riding income $E$ and $F$ obtained by manufacturers and influencers when adopting conservative strategies are both 7 . When consumers actively purchase products, the amount of input cost reduction $J$ is 30, and the cost $H$ for consumers to reward influencers is 18 . When both manufacturers and influencers choose a conservative strategy, the compensation $W$ and $K$ that need to be paid to each other are both 10 . The discount $\theta$ for consumers who choose a conservative strategy is 50 per cent. The proportion coefficients $m$ and $n$ of the valueadded income obtained by manufacturers and the input cost is 80 per cent. The proportion coefficient of influencers is 20 per cent.

This paper takes this simulation experiment as an example and analyses each participant's game evolution process in livestreaming e-commerce. It provides decision-making assistance for the participants of the livestreaming e-commerce model in the real world. Through the above analysis of the equilibrium point stability of the evolutionary game, it can be seen that the decision-making behavior of manufacturers, social media influencers, and consumers is closely related to the income and expenditure of all participants. This paper uses Matlab simulation software to simulate and analyze the above game models to analyze the stable evolution process of strategy selections of manufacturers, influencers, and consumers under different probabilities. It discusses the initial probability available to participants, active participation behavior and reward behavior of consumers, and the distribution coefficient of income and expenditure based on simulation results. 
4.1. The Impact of Different Initial Probabilities on the Stable Evolution of the Strategy Selection of Each Participant in Livestreaming E-commerce

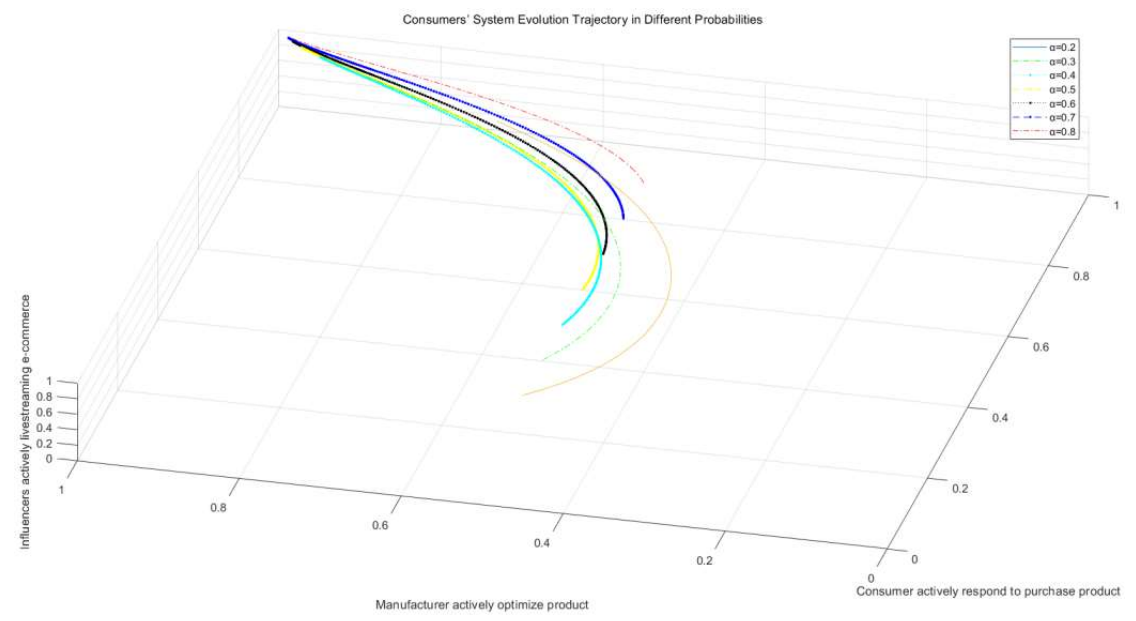

Figure 2. The systematic evolution trajectory of consumers in different response probabilities.

When the probabilities of consumers actively responding to social media influencers' calls are $0.2,0.3,0.4,0.5,0.6,0.7,0.8$, the following conclusions can be drawn based on the simulation results in Figure. 2: (1) Based on the assumptions of "economic man" or "rational man", since the probability that consumers actively responding to influencers' calls to purchase products is positively correlated with the discount given to consumers in livestreaming e-commerce, the probability of the consumers' response to livestreaming ecommerce is mostly based on their interests and demands. Consumers select the decision that tends to maximize their interests by measuring the discount they get. (2) As the probability of consumers actively responding to influencers' calls to purchase products increases, $\alpha, \beta, \gamma$ converge to 1 . The final equilibrium point tends to be $(1,1,1)$. That is, all three participants in the game finally choose to actively respond to purchase products, actively optimize products, and actively carry out livestreaming e-commerce as the final strategies. At this point, the increase in the probability of consumers' active response promotes manufacturers and social media influencers to choose active strategies. Consumers greatly influence the probability of influencers choosing active strategies. (3) In the process that the probability of consumers actively responding to influencers' calls to purchase products increases from 0.2 to 0.5 , the increase in the probability of manufacturers and influencers choosing active strategies is greater than that of consumers actively responding to influencers' calls to purchase products, which is from 0.5 to 0.8 . Combining with the principle of diminishing marginal effects, it can be seen that in a low discount, consumers get benefits from the beginning of no discount, the tendency for profit leads to a significant increase in the probability that consumers choose to actively respond to influencers' calls. When the discount given by living commerce reaches some levels, consumers are less sensitive to discount, which makes the probability of consumers choosing to actively respond to influencers' calls to purchase products relatively flat. Therefore, influencers should choose to fully integrate the characteristics of consumers under a not too high discount limit to form a better development situation to achieve a win-win situation with manufacturers. 


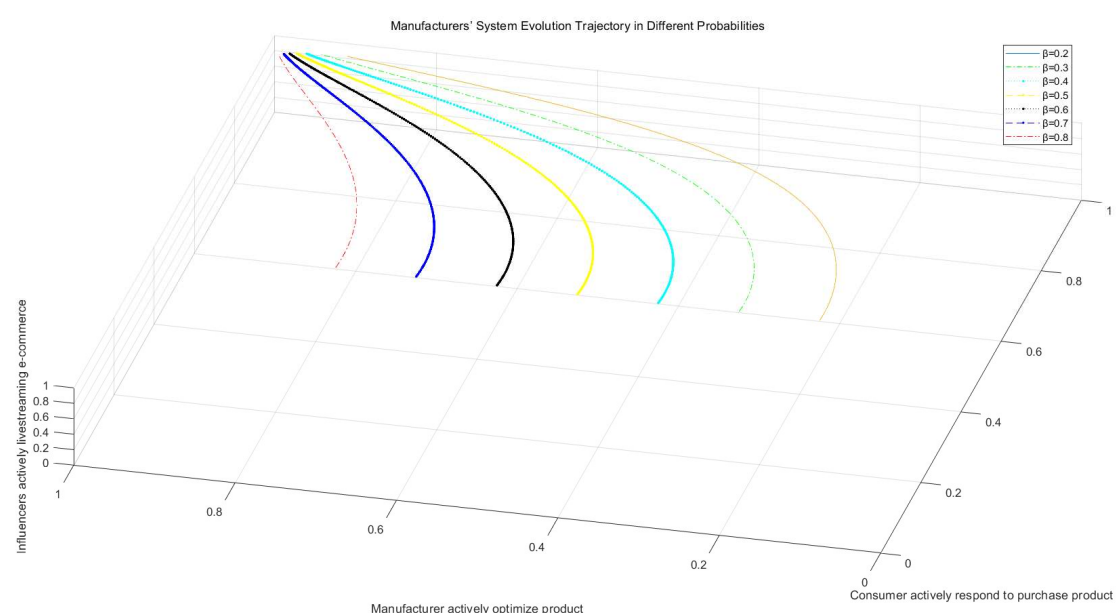

Figure 3. The systematic evolution trajectory of manufacturers choosing to actively optimize products in different probabilities.

When manufacturers' probabilities to actively optimize products are $0.2,0.3,0.4,0.5$, $0.6,0.7$ and 0.8 , the simulation will be performed. The evolution process is shown in Figure. 3: (1) As the probability of manufacturers actively optimizing products increases, $\alpha, \beta, \gamma$ converge to 1 . The final equilibrium point tends to be $(1,1,1)$. All three participants in the game in the supply chain finally choose to actively respond to purchase products, actively optimize products, and actively carry out livestreaming e-commerce as the final strategies. At this point, the increase in the probability of manufacturers actively optimizing products promotes both consumers and social media influencers to choose an active strategy, and manufacturers greatly influence the probability of influencers choosing an active strategy. (2) When the probability of manufacturers choosing to actively optimize products strategy is low $(\beta=0.1,0.3)$, the probability of consumers actively responding to influencers' calls to purchase products increases slowly. Although influencers choose to actively carry out livestreaming e-commerce, it is difficult for consumers to actively respond to purchase products. (3) When manufacturers choose to actively optimize products, it can effectively enhance the enthusiasm of influencers actively carrying out livestreaming e-commerce. Manufacturers were choosing an active strategy prompt influencers to voluntarily choose an active livestreaming e-commerce strategy from their profitability in the era of informatization. Manufacturers need to understand consumers' intention and market orientation in real-time with big data and adjust their business activities in time. In this case, social media influencers tend to choose an active livestreaming ecommerce strategy when manufacturers adopt an active strategy, enabling consumers who participate in livestreaming e-commerce to obtain more discounts. To sum up, manufacturers' strategy of actively optimizing products can effectively promote influencers to choose an active livestreaming e-commerce strategy, thereby driving consumers to actively participate in livestreaming e-commerce. 


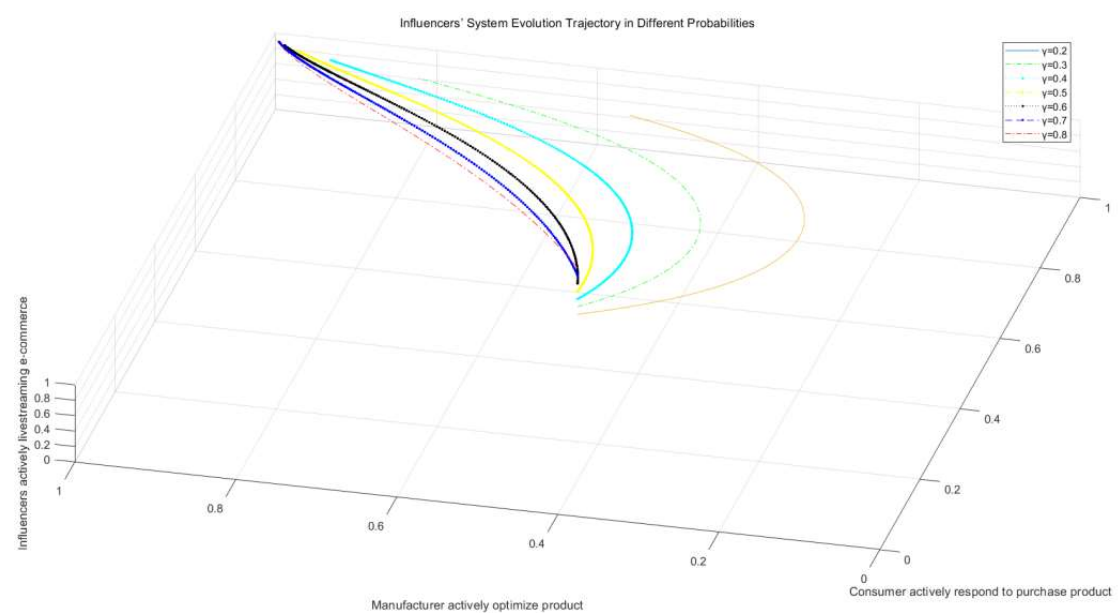

Figure 4. The systematic evolution trajectory of social media influencers choosing to carry out livestreaming e-commerce actively in different probabilities.

When the probabilities of social media influencers actively carrying out livestreaming e-commerce are $0.2,0.3,0.4,0.5,0.6,0.7,0.8$, the simulation will be performed. It can be seen from the simulation results in Figure 4: (1) As the probability of influencers actively carrying out livestreaming e-commerce increases, $\alpha, \beta, \gamma$ converge to 1 , and the final equilibrium point tends to be $(1,1,1)$. All three participants in the game in the supply chain finally choose to actively respond to purchase products, actively optimize products, and actively carry out livestreaming e-commerce as the final strategies. At this point, the increase in the probability of influencers actively carrying out livestreaming e-commerce promotes both consumers and manufacturers to choose an active strategy. Influencers greatly influence the probability of manufacturers choosing an active strategy. (2) Social media influencers' actively carrying out livestreaming e-commerce strategy increase the probability that manufacturers choose to optimize products since manufacturers are the participant for profit. Manufacturers' choice of business activities needs to consider income and expenditure. When the probability of social media influencers choosing an active livestreaming e-commerce strategy increases, if manufacturers also adopt the strategy of actively optimizing products, more consumers can participate in livestreaming e-commerce purchase products. (3) In addition, when the probability of influencers choosing an active livestreaming e-commerce strategy is too high $(\gamma=0.7,0.8)$, the probability of consumers actively responding to influencers' calls to purchase products changes very little. It can be seen that the probability that influencers were choosing an active livestreaming e-commerce strategy is not positively correlated to the probability that consumers were actively responding to influencers' calls to purchase products. When the enthusiasm of influencers when carrying out livestreaming e-commerce exceeds any limit, the impact on consumers' choices of actively responding to influencers' calls to purchase products is not obvious. 
4.2. The Impact of Consumers' Participation and Reward Behavior on the Stable Evolution of the Strategy Selection of Each Participant in Livestreaming E-commerce

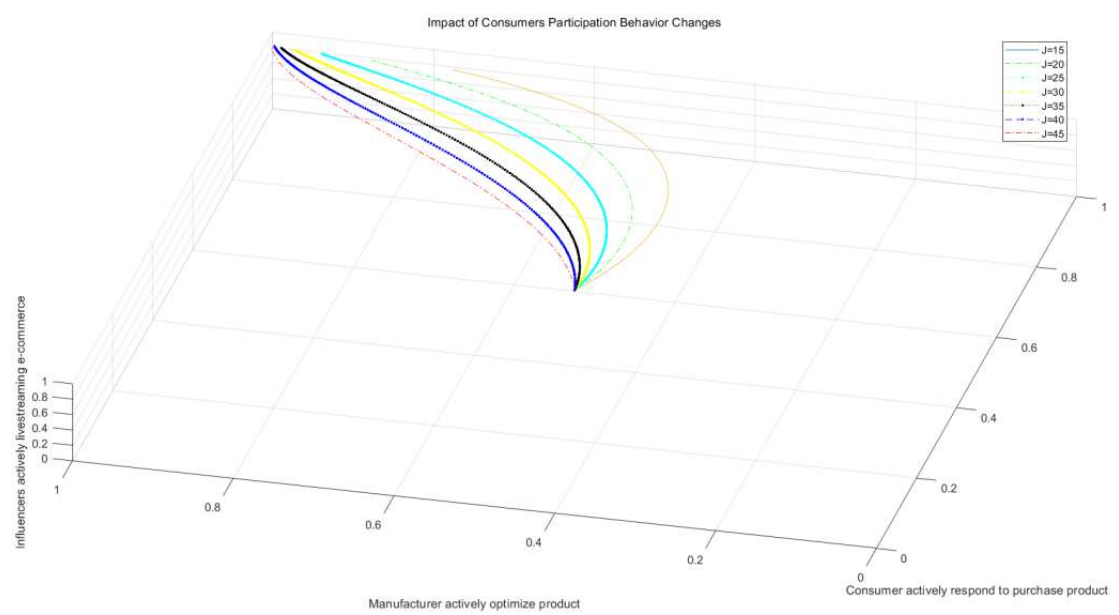

Figure 5. The systematic evolution trajectory of consumers participation behavior changes.

Consumers' participation in livestreaming e-commerce is mainly reflected in two aspects: one is to actively respond to social media influencers' calls and participate in livestreaming e-commerce to purchase products, which will reduce the cost of manufacturers and influencers. The other is to reward influencers. That is, consumers directly reward money to influencers instead of manufacturers through livestreaming platforms. Figure 5 is a simulation of the impact of changes in input cost $J$ reduced by consumers' actively participating in livestreaming e-commerce to purchase products when other parameters remain unchanged. According to the simulation results: (1) The change in input cost reduction $J$ brought by consumers' participation will influence the decision-making of manufacturers and influencers. The greater the reduction in input cost, the higher the probability of manufacturers and influencers choosing an active livestreaming strategy. (2) The change in cost reduction has a greater impact on the probability of manufacturers optimizing products. Since when consumers actively respond to social media influencers' calls to purchase products, consumers will provide certain behavioral support to manufacturers and influencers, thereby reducing the input cost of livestreaming e-commerce and bringing more benefits to manufacturers and influencers. (3) Since manufacturers have a greater apportionment ratio of income and expenditure, manufacturers are more sensitive to changes of interests than influencers, making the impact of changes in cost reduction $J$ on strategy selection of manufacturers more significant.

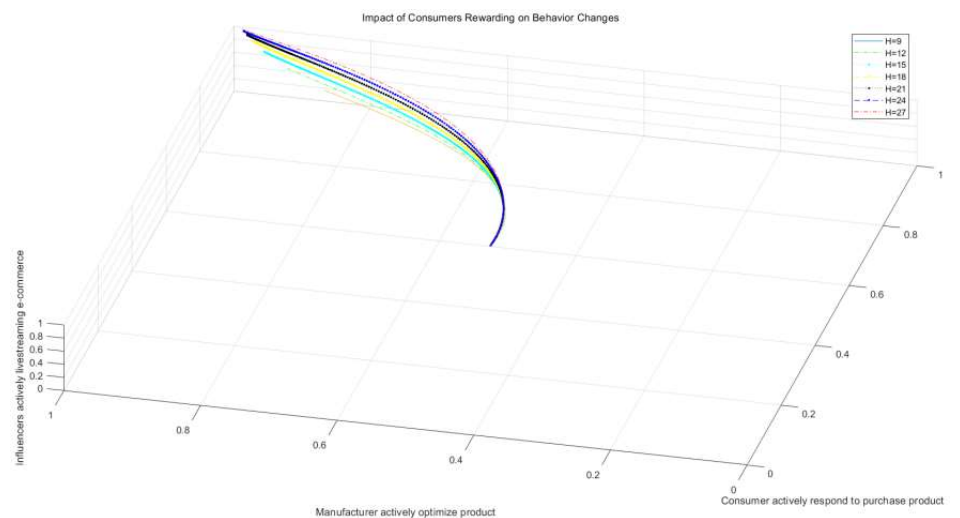

Figure 6. The systematic evolution trajectory of consumers rewarding behavior changes. 
Figure 6 is a simulation of the impact of changes in consumers' reward amount $H$ for social media influencers on the game strategies of manufacturers and influencers when other parameters remain unchanged. According to the simulation results: (1) With the increase of consumers' reward, the probability of influencers choosing an active livestreaming e-commerce strategy has gradually increased. (2) There is almost no change in the probability of manufacturers actively optimizing products since consumers' reward is mostly given to influencers directly through livestreaming platforms, and the reward is mostly irrelevant to manufacturers. Changes in consumers' reward $\mathrm{H}$ have a more significant impact on the strategy selection of influencers.

\subsection{The Impact of Distribution Coefficient on the Stable Evolution of the Strategy Selection of Each Participant in Livestreaming E-commerce}

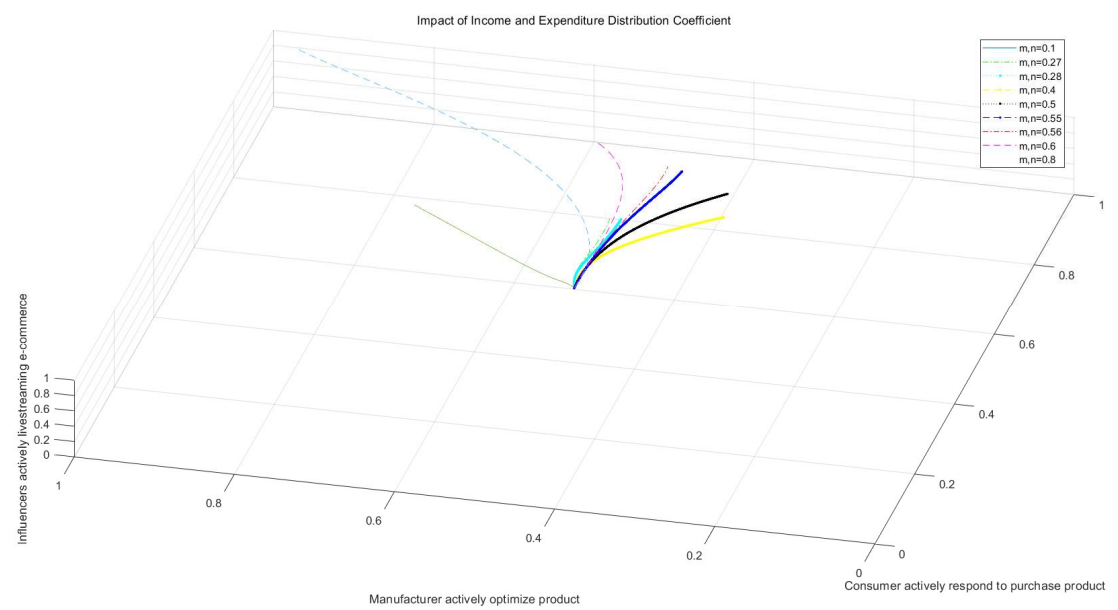

Figure 7. The systematic evolution trajectory of distribution coefficient changes.

Figure 7 is a simulation of the impact of changes in income and expenditure distribution coefficients $m$ and $n$ of manufacturers and social media influencers on the game strategy when other parameters remain unchanged. The simulation results depict two critical values for the apportionment ratio, which are between $0.55 \sim 0.56$ and $0.27 \sim 0.28$. At this point, there are three kinds of game decision-making: (1) When the distribution coefficients $m$ and $n$ are higher than 0.56 , the enthusiasm of manufacturers and influencers to promote livestreaming e-commerce gradually increases, $\beta$ and $\gamma$ converge to 1 . The final equilibrium point tends to be $(1,1,1)$. At this point, both manufacturers and influencers choose an active livestreaming e-commerce strategy. (2) When $m$ and $n$ are between $0.28 \sim 0.55$, the enthusiasm of manufacturers and influencers to promote livestreaming ecommerce gradually decreases, $\beta$ and $\gamma$ converge to 0 , and the final equilibrium point tends to be $(1,0,0)$. At this time, both manufacturers and influencers choose a conservative livestreaming e-commerce strategy. (3) When $m$ and $n$ are lower than 0.27 , the probability of manufacturers choosing to optimize products increases, while the probability of influencers choosing an active livestreaming e-commerce strategy decreases, $\beta$ converges to $1, \gamma$ converges to 0 , and the final equilibrium point tends to be $(1,1,0)$. Influencers are unwilling to bear too much cost, which reduces the probability of influencers choosing an active livestreaming e-commerce strategy. Therefore, social media influencers eventually tend to choose a conservative livestreaming e-commerce strategy; simultaneously, manufacturers pay only a small cost to get a lot of income, so they are more inclined to choose to actively optimize products. In summary, it can be seen that controlling the income and expenditure distribution coefficient above 0.56 is more in line with the interests of each participant and can promote the development of the livestreaming e-commerce model. 


\section{Conclusions}

According to the forecast of EqualOcean Intelligence, livestreaming e-commerce is expected to reach 2.8 trillion yuan in 2024, and the penetration rate is close to $15 \%$ [37]. In other words, livestreaming e-commerce isn't a short-lived phenomenon, but the transformation and upgrading of the e-commerce industry in compliance with the regulation of consumption upgrade and informatization. With the continuous improvement of the standardization of livestreaming e-commerce, its impact on industrial transformation, economic empowerment and society will continue to increase [38]. This paper combines the three-party game with livestreaming e-commerce, tests the income and expenditure of livestreaming e-commerce by experiments. Our results provide a new perspective on the evolution of all participants in livestreaming e-commerce and helping livestreaming e-commerce participants to understand the changes in their income:

- Based on the premise of the bounded rationality of game participants, this paper adopts the evolutionary game to establish a three-party game income and expenditure matrix involving manufacturers, social media influencers, and consumers. It systematically analyzes the decision-making evolution of each participant in the live commerce supply chain, and combined with simulation analysis, the game strategies and influencing factors of social media influencers manufacturers and consumers were investigated;

- The strategies selected by manufacturers, social media influencers, and consumers have different impacts on each other's decision-making mechanisms. To better promote livestreaming e-commerce and enhance the interests of each participant, consumers can subsidize influencers by gift-giving to increase their enthusiasm for livestreaming e-commerce. Manufacturers should take the initiative to adjust their business activities according to the market trends and select products that satisfy market needs to participate in livestreaming e-commerce. Influencers should maintain enthusiasm to improve the quality of livestreaming e-commerce to promote the overall income of livestreaming e-commerce;

- Manufacturers are more sensitive to consumers' participation, and social media influencers are more sensitive to consumers' reward. Consumers can actively participate in livestreaming e-commerce while enjoying the discount, providing more support for manufacturers and influencers, and achieving mutual benefit and win-win results;

- Manufacturers are more sensitive to the distribution coefficient of income and expenditure than social media influencers, so it's necessary to formulate a reasonable income and expenditure distribution mechanism to optimize the enthusiasm of manufacturers and influencers to promote livestreaming e-commerce. When setting the ratio of income and expenditure distribution of livestreaming e-commerce, the proportion of manufacturers is generally higher to ensure the expected enthusiasm of manufacturers and influencers.

However, there is not much research on livestreaming e-commerce, especially the lack of actual data. This paper only uses simulated data for simulation experiments and lack actual data reference. At the same time, this paper mainly researches the income and expenditure of the agricultural product supply chain in livestreaming e-commerce. The income and expenditure of different goods in livestreaming e-commerce may be different, which needs to be further explored. Future researchers can combine actual data to further examine the supply chain's decision-making mechanism in livestreaming e-commerce and discuss the income and expenditure of high-end luxury goods, electronic equipment, books, or other products in livestreaming e-commerce. Furthermore, we will try to explore the specific impact of the livestreaming e-commerce model in different aspects such as society or market, especially in the big data context $[39,40]$, to verify and improve its scalability and universality. 
Author Contributions: Conceptualization, Jixiao Wu and Yinghui Wang; methodology, Jixiao Wu; software, Jixiao $\mathrm{Wu}$; formal analysis, Jixiao $\mathrm{Wu}$.; investigation, Jixiao $\mathrm{Wu}$; writing - original draft preparation, Jixiao $\mathrm{Wu}$; writing - review and editing, Jixiao $\mathrm{Wu}$ and Yinghui Wang; funding acquisition, Yinghui Wang. All authors have read and agreed to the published version of the manuscript.

Funding: This research was funded by Hebei Provincial Department of Education (Project number SR 201913); and the National Social Science Fund of China (Project number 16FGL003).

Data Availability Statement: Not applicable.

Acknowledgments: In this section, you can acknowledge any support given which is not covered by the author contribution or funding sections. This may include administrative and technical support, or donations in kind (e.g., materials used for experiments).

Conflicts of Interest: The authors declare no conflict of interest.

\section{References}

1. Li Xiangrong; Zhu Shaoying. Cause Analysis and Countermeasures of the Chaos in the Webcast. People's Tribune, 2020. 19: p. 6465.

2. Li Yilun. Analysis on the Development Model of Integration of "Internet Celebrity Economy" and Publishing. View on Publishing, 2020. 17: p. 40-42.

3. Sjöblom, M.; et al.. Content structure is king: An empirical study on gratifications, game genres and content type on Twitch. Computers in Human Behavior, 2017. 73: p. 161-171.

4. Sjöblom, M.; J. Hamari. Why do people watch others play video games? An empirical study on the motivations of Twitch users. Computers in Human Behavior, 2017. 75: p. 985-996.

5. Tobon, S.; J. García-Madariaga. The Influence of Opinion Leaders' eWOM on Online Consumer Decisions: A Study on Social Influence. Journal of Theoretical and Applied Electronic Commerce Research, 2021. 16(4): p. 748-767.

6. Yu, E.; et al.. Impact of viewer engagement on gift-giving in live video streaming. Telematics and Informatics, 2018. 35(5): p. 14501460.

7. Hu, M.; S.S. Chaudhry. Enhancing consumer engagement in e-commerce live streaming via relational bonds. Internet Research, 2020. 30(3).

8. Su, J.; et al.. An Intelligent Method for Lead User Identification in Customer Collaborative Product Innovation. Journal of Theoretical and Applied Electronic Commerce Research, 2021. 16(5): p. 1571-1583.

9. Zhao, K.; et al.. Direct and Indirect Spillovers from Content Providers' Switching: Evidence from Online Live Streaming. 2019.

10. Cunningham, S.; D. Craig; J. Lv. China's livestreaming industry: platforms, politics, and precarity. International Journal of Cultural Studies, 2019. 22(6).

11. Alp, Z.Z.; Ş.G. Öğüdücü. Identifying topical influencers on twitter based on user behavior and network topology. Knowledge-Based Systems, 2018. 141.

12. Wongkitrungrueng, A.; N. Assarut. The role of live streaming in building consumer trust and engagement with social commerce sellers. Journal of Business Research, 2020. 117(prepublish).

13. Wongkitrungrueng; Dehouche; Assarut. Live streaming commerce from the sellers' perspective: implications for online relationship marketing. Journal of Marketing Management, 2020. 36(5-6).

14. Fei, M.; et al.. Promoting or attenuating? An eye-tracking study on the role of social cues in e-commerce livestreaming. Decision Support Systems, 2021. 142.

15. Lee, E.-J.; J. Overby.Creating value for online shoppers: Implications for satisfaction and loyalty. Journal of Consumer Satisfaction, 2004. 17: p. 54-67.

16. Cai, J.; et al.. Utilitarian and Hedonic Motivations for Live Streaming Shopping. Interactive Experiences for TV and Online Video, 2018. 
17. Luo Guihua. Research on the Application of Network Broadcast in the Development of Rural E-commerce. Postgraduate, Central South University of Forestry and Technology, Hunan, 2019.

18. Yang, C.; et al.. Online User Review Analysis for Product Evaluation and Improvement. Journal of Theoretical and Applied Electronic Commerce Research, 2021. 16(5): p. 1598-1611.

19. Liu Zhongyu; Zhao Xianghao; Long Wei. The Formation Mechanism of Consumers' Purchase Intention under the Influencer Marketing - - An Analysis based on Grounded Theory. China Business and Market, 2020. 34(8): p. 48-57.

20. Bhattacharyya, S.; I. Bose. S-commerce: Influence of Facebook likes on purchases and recommendations on a linked e-commerce site. Decision Support Systems, 2020. 138.

21. Meng Yanbei. Research on the Regulation of Anchor's Commercial Propaganda Behavior in Live Delivery. People's Tribune, 2020. 25,: p. 116-119.

22. Sun, Y.; et al.. How live streaming influences purchase intentions in social commerce: An IT affordance perspective. Electronic Commerce Research and Applications, 2019. 37.

23. Friedman, D.. Evolutionary Games in Economics. Econometrica, 1991. 59(3): p. 637-666.

24. Zhang Huimei; Deng Zigang. Analysis of Corporate Social Responsibility Based on Three-side Dynamic Game between Government, Society and Enterprises. Systems Engineering, 2020. 29(6): p. 123-126.

25. Kang, K.; et al.. The dynamic effect of interactivity on customer engagement behavior through tie strength: Evidence from live streaming commerce platforms. International Journal of Information Management, 2021. 56: p. 102251.

26. Huang Xiongkun. Game Analysis on "E-business Companies+ Peasant Household" Model for Fresh Products. Postgraduate, Jiangxi University of Finance \& Economics, Jiangxi, 2019.

27. Wen Zongchuan; Wu Xingyang. Research on Coodinated Development of Main-body Elements of Rural E-commerce Logistics. Journal of Beijing Jiaotong University (Social Sciences Edition), 2020. 19(5): p. 118-126.

28. Zhang Cheng; Zhang Guangsheng; Wang Yanling. Co-evolution and Policy Optimization of Rural E-commerce and Rural Logistics under Government Poverty Alleviation. Journal of Beijing Jiaotong University (Social Sciences Edition), 2020. 19(1): p. 98-105.

29. Yi, Z.; et al.. Evolutionary game analysis and simulation with system dynamics for behavioral strategies of participants in crowd logistics. Transportation Letters-the International Journal of Transportation Research, 2020.

30. Chen Fuji, Huang Jiangling. The Strategy of Managing Net-cheaters Based on Three-side Game. Chinese Public Administration, 2013. 11: p. 18-21.

31. Yang, D.; et al.. Trilateral Game Aided Information Management for Open Complex Giant Systems, in The 6th International Conference on Communications, Signal Processing, and Systems (CSPS). 2017.

32. Yu Qijia. Game Analysis of Fresh Agricultural Products Supply Chain Subjects under Online Purchase Mode. Postgraduate, Harbin University of Commerce, Harbin, 2019.

33. Wu Jie; Che Xiaojing; Sheng Yongxiang; et al. Study on Government-industry-unibersity-insititute Collaborative Innovation Based on Tripartite Evolutionary Game. Chinese Journal of Management Science, 2019. 27(1): p. 162-173.

34. Su, L.; et al.. Impact of E-Commerce Adoption on Farmers' Participation in the Digital Financial Market: Evidence from Rural China. Journal of Theoretical and Applied Electronic Commerce Research, 2021. 16(5): p. 1434-1457.

35. Lin, J.; et al.. How do agribusinesses thrive through complexity? The pivotal role of e-commerce capability and business agility. Decision Support Systems, 2020. 135.

36. Li Yabing; Zhang Jiarui. Evolutionary Game of Webcast Governance Strategy: Stakeholder Perspective. Economy and Management, 2020. 34(2): p. 25-31.

37. Niu Zekun; Wei Fan. The Logic of "Bring Goods": Research Report on the Industry Chain of Live Commerce. EqualOcean Intelligence, Beijing, 2020. 
38. Gyenge, B.; et al.. A New Strategic Marketing Management Model for the Specificities of E-Commerce in the Supply Chain. Journal of Theoretical and Applied Electronic Commerce Research, 2021. 16(4): p. 1136-1149.

39. Puka, R.; S. Jedrusik. A New Measure of Complementarity in Market Basket Data. Journal of Theoretical and Applied Electronic Commerce Research, 2021. 16(4): p. 670-681.

40. Zong, K.; et al.. Or-Based Intelligent Decision Support System for E-Commerce. Journal of Theoretical and Applied Electronic Commerce Research, 2021. 16(4): p. 1150-1164. 